\title{
Cost and cost-effectiveness of adjuvant trastuzumab in the real world setting: A study of the Southeast Netherlands Breast Cancer Consortium
}

\author{
Shanly C. Seferina ${ }^{1,2}$, Bram L.T. Ramaekers ${ }^{8}$, Maaike de Boer $^{1,2}$, M. Wouter \\ Dercksen $^{3}$, Franchette van den Berkmortel ${ }^{4}$, Roel J.W. van Kampen ${ }^{1,5}$, Agnès J. \\ van de Wouw ${ }^{6}$, Adri C. Voogd ${ }^{1,2,7}$, Vivianne C.G. Tjan Heijnen ${ }^{1,2}$, Manuela A. Joore ${ }^{8,9}$ \\ ${ }^{1}$ Department of Medical Oncology, Maastricht University Medical Center, Maastricht, The Netherlands \\ ${ }^{2}$ GROW-School for Oncology and Developmental Biology, Maastricht University Medical Center, Maastricht, The Netherlands \\ ${ }^{3}$ Department of Internal Medicine, Máxima Medical Center, Veldhoven, The Netherlands \\ 4 Department of Internal Medicine, Zuyderland Medical Center, Heerlen, The Netherlands \\ ${ }^{5}$ Department of Internal Medicine, Zuyderland Medical Center, Sittard-Geleen, The Netherlands \\ ${ }^{6}$ Department of Internal Medicine, VieCuri Medical Center, Venlo, The Netherlands \\ 7 Department of Epidemiology, Maastricht University Medical Center, Maastricht, The Netherlands \\ ${ }^{8}$ Department of Clinical Epidemiology and Medical Technology Assessment, Maastricht University Medical Center, Maastricht, \\ The Netherlands \\ ${ }^{9}$ School for Public Health and Primary Care, Maastricht University Medical Center, The Netherlands \\ Correspondence to: Manuela A. Joore, email: m.joore@mumc.nl
}

Keywords: trastuzumab, cost-effectiveness, early breast cancer, real-world, Markov model

Received: January 23, $2017 \quad$ Accepted: March 10, $2017 \quad$ Published: April 09, 2017

Copyright: Seferina et al. This is an open-access article distributed under the terms of the Creative Commons Attribution License 3.0 (CC BY 3.0), which permits unrestricted use, distribution, and reproduction in any medium, provided the original author and source are credited.

\section{ABSTRACT}

Background: We assessed the real world costs and cost-effectiveness of the addition of trastuzumab in HER2 positive early breast cancer compared to chemotherapy alone in the Dutch daily practice as opposed to the results based on trial data and based on a subset of patients that were treated according to the guidelines.

Patients and Methods: In a cohort study, we included all patients with stage I-III invasive breast cancer treated with curative intent in 5 Dutch hospitals between 2005 and $2007(n=2684)$.We assessed three scenarios: a real-world scenario, a trial scenario and a guideline scenario, with costs and effectiveness based on either the cohort study, the published trials or the guidelines. Incremental cost-effectiveness ratios (ICERs) and cost-effectiveness acceptability curves (CEACs) were constructed.

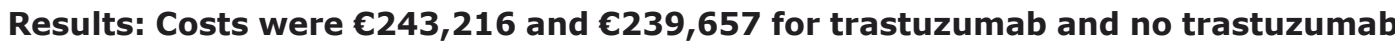

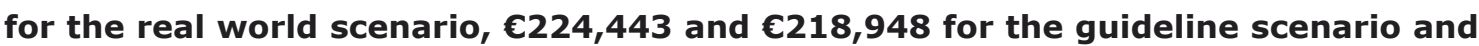
$C 253,666$ and $C 265,116$ for the trial scenario. The QALYs were $0.827,0.861,0.993$ for the real world, guideline and trial scenario. The corresponding ICERs were

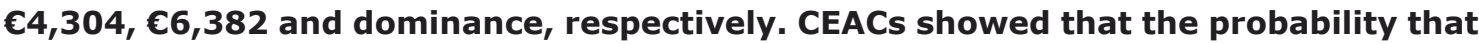
trastuzumab is cost-effective is $\geq 99 \%$ in each scenario.

Conclusion: Adjuvant trastuzumab in the real world can be considered costeffective.

\section{INTRODUCTION}

In the Netherlands, the use of trastuzumab for the adjuvant treatment of patients with HER2-positive early breast cancer was estimated to be $€ 5,828$ per quality adjusted life year (QALY) gained (using a health care perspective). We obtained this Dutch estimate of the cost-effectiveness by transferring the results of a UK 
(commissioned by NICE) model-based cost-effectiveness analysis of trastuzumab in the early breast cancer setting to the Dutch setting. [1] This analysis provided an early indication of the cost-effectiveness of trastuzumab in the adjuvant setting in the Netherlands. Partly based on this analysis, the Dutch Health Care Insurance Board (CVZ) permitted provisional reimbursement of trastuzumab as adjuvant treatment as of 2005. However, although the structure of the UK cost-effectiveness model was deemed transferable, the model inputs appeared to be only partially transferable to the Dutch setting. Continued reimbursement depended on study of prospective data on the cost-effective use of trastuzumab in daily practice within the Netherlands. A number of studies have investigated the cost-effectiveness of trastuzumab in the adjuvant setting. These studies typically found that adjuvant trastuzumab treatment has an acceptable cost-effectiveness ratio. [2-7] However, in most cases estimates of resource use were based on small datasets or even expert opinion. Moreover, these studies were based on the assumption that the effectiveness and, to some extent, resource use in the real world are equal to the ones observed in a trial setting. Since it is known that randomized controlled trials have a strong internal validity but may have limited external validity, there is reason to think that the real world indication (unselected population) and treatment regimens for trastuzumab differ from the trial situations (selected population) and that the effectiveness is likely to be different too. [8].

The primary aim of our analysis was to assess the costs and cost-effectiveness of one year of adjuvant trastuzumab compared to no trastuzumab for HER2 positive breast cancer, given sequentially after chemotherapy in clinical practice (real world) as opposed to the cost-effectiveness of trastuzumab based on trial data and based on a subset of patients that were treated in full concordance with the Dutch clinical guideline.

\section{PATIENT AND METHODS}

\section{Model description}

In this study we assessed the costs and costeffectiveness of one year of adjuvant trastuzumab treatment in early HER2-positive breast cancer versus chemotherapy alone. A Markov health state transition cohort model was developed for HER2 positive breast cancer consisting of four health states: (1) disease-free survival (DFS), (2) locoregional recurrence, contralateral recurrence and a new primary tumor (local recurrence, LR), (3) distant metastases (DM), and (4) death (due to cancer and due to other causes). Additionally, in the model cardiac toxicity could occur during the first year (Figure 1). The time horizon of the model was lifetime and the cycle length one year. The analyses were conducted using the Dutch health care perspective.

Transitions in the model were estimated using an exponential survival function (based on Kaplan Meier (KM) curves) for overall survival (OS), DFS and DM. These survival functions were used to calculate the annual probabilities of LR, DM and mortality. The model was used to compare the costs, life years (LYs) and QALYs of treatment with one year of adjuvant trastuzumab sequentially after chemotherapy in patients with HER2positive early breast cancer versus chemotherapy alone. We assessed how the incremental costs of trastuzumab related to differences in LYs and QALYs.

Three scenario analyses were performed, a real world scenario, a trial scenario and a guideline scenario. In all three scenarios, the relative treatment effect of trastuzumab was based on the HERA trial. In addition, in all three scenarios health care resource use other than trastuzumab costs were based on the real world cohort study. The scenarios differed in how the annual probabilities for trastuzumab (OS, DFS, DM-free survival and cardiac toxicity) and the costs of trastuzumab (i.e. number of administrations and dose per patient) were determined.

In the real world scenario annual trastuzumab probabilities for OS, DFS, DM-free survival and cardiac toxicity, and costs associated with trastuzumab were taken from all patients who received trastuzumab in the real world cohort study. [9].

In the guideline scenario annual probabilities for OS, DFS, DM-free survival and cardiac toxicity, and costs associated with trastuzumab were taken from the subgroup of patients in the real world cohort study who received trastuzumab following the criteria listed in the 2005 Dutch clinical guideline. [9].

In the trial scenario in which annual probabilities for OS, DFS, DM-free survival and cardiac toxicity were taken from the HERA trial, and costs associated with trastuzumab were partly taken from the HERA trial and partly from the real world cohort study. [10-13].

The model was built and analyzed in Microsoft Excel. An overview of the sources of the input parameters in the three scenario analyses is provided in Table 1.

\section{Model input parameters}

\section{Real world cohort study}

Detailed information on patient and tumor characteristics are reported elsewhere. [9].

In brief, we included 2684 patients with consecutive diagnosis of stage I-III breast cancer in the five participating hospitals between January, 1st 2005 and December 31st, 2007. Follow-up was collected until October 20th 2011. Data were collected by trained data managers under direct supervision of medical specialists 
and researchers. For patients of whom the HER2 status was missing, central pathology review was performed. In total, 476 patients had a HER2 positive tumor. Patients were classified in a cohort of patients who received trastuzumab $(n=230)$ and a cohort of patients who did not receive trastuzumab $(n=246)$. In the guideline scenario there were 196 patient who did received trastuzumab and 191 patients who did not receive trastuzumab.

\section{Transition probabilities}

In the real world scenario, transition probabilities were estimated based on the trastuzumab group in the cohort study. Hazard ratios (HR) were taken from the HERA trial, and applied to the real world exponential survival functions for 8 years, afterwards the HRs were assumed to be 1 . HRs for DFS and OS were based on the 8-years follow up data of the HERA trial [13]. The HR for DM-free survival was based on the 2-years follow up data [11] as for this outcome 8-years follow up was not reported. The relative risk (RR) for cardiac toxicity was based on the HERA trial. [14] In the scenario based on the 2005 guideline the same approach was taken, using the subset of patients $(\mathrm{N}=196)$ who received trastuzumab following the Dutch 2005 clinical guideline to derive the transition probabilities. In the trial scenario DFS and OS were based on the 8-years follow up data of both arms of the HERA trial [13]. DM was based on the 2-years follow up data [11] as for this outcome 8-years follow up was not reported. Age related mortality based on Dutch allcause mortality for women was used to estimate the timedependent mortality probability for the DFS health state in all three scenarios. [15].

\section{Resource use and costs}

Health care resource use was based on the real world cohort study. The trastuzumab related costs included trastuzumab treatment, hospital cost when receiving the treatment and cardiac monitoring costs. Also the cost to determine the HER2 status was included, using either immunohistochemistry (IHC) tests, FISH tests, or both. The actual amount of trastuzumab vials used was determined for the calculation of drug costs (taking into account drug spill). The number of cycles, used to calculate the drug costs and costs of administration, was either based on cohort data (real-world and guideline scenario) or based on the HERA trial (trial scenario).

We assumed all other medical resource use was independent from the use of trastuzumab. The medical costs per health state were based on the total hospital resource use as observed among all HER2 positive patients in the real world cohort study. This included surgical procedures, diagnostic procedures, radiotherapy, pharmacological treatments, admissions, outpatient consultations. These were estimated separately for patients with DFS, LR, and DM and separately for the first year and subsequent years. Unit prices for drugs were based on the Dutch reimbursement system for pharmaceuticals. [16]
All other unit costs were derived from the national health tariffs authority. [17] Indexation to 2012 price levels was carried out using price index numbers reported by the Central Bureau of Statistics (CBS) in the Netherlands. [15] Total costs per health state are shown in Table 3.

\section{Health state utilities}

Quality of life weights were derived from a cross sectional survey among patients with breast cancer in four of the five medical centers participating in the real world cohort study $(n=268)$. At their regular outpatient visit patients with breast cancer, irrespective of treatment or disease state, were asked to fill out the EQ-5D questionnaire once. The health state utility scores were calculated using the UK tariff developed by Dolan et al. [18] Severe cardiac adverse events were not observed in the patients who participated in the cross sectional survey. Therefore, a utility for symptomatic heart failure (0.600; [19]) was taken from the literature to calculate a disutility of $0.128(=0.728-0.600)$ for severe cardiac adverse events. The utility 0.728 is the mean utility for DFS in the first year after diagnosis from the cohort. See Table 3 for an overview of the utility scores used in the model.

\section{Analyses}

\section{Base case analyses}

Expected LYs, QALYs and costs (cardiac event/ monitoring cost, disease free cost, local recurrence cost and distant metastases cost) were estimated in the three scenarios. Subsequently, the incremental cost-effectiveness ratio (ICER) for each scenario was calculated by dividing the incremental costs by the incremental QALYs. If the ICER is below the maximum amount society is willing to pay per gained QALY, the new treatment can be considered cost-effective. In the Netherlands, a maximum willingness-to-pay threshold of 80,000 euro per gained QALY was proposed for high burden diseases. [20] A half cycle correction was applied for QALYs and health state costs in all three scenarios. Discounting was carried out according to the Dutch guidelines for pharmacoeconomic research, in which future costs and effects are discounted by rates of $4.0 \%$ and $1.5 \%$, respectively. [17].

\section{One-way sensitivity analysis}

Although the 8-years results of the HERA trial still showed a treatment benefit, the impact of a shorter duration of treatment benefit of 4 years was explored in a one-way sensitivity analysis. [13] Moreover, the impact of assuming treatment dependent DM health state cost (instead of treatment independent as in the base case analysis) was explored. Resulting in lower cost for patients treated with trastuzumab versus those not treated with trastuzumab. 
Probabilistic sensitivity analysis and value of information analysis

Probabilistic sensitivity analysis (PSA) was performed to examine the uncertainty in all stochastic input parameters simultaneously. This was done by assigning distributions to the input parameters and drawing random values from these distributions using Monte Carlo simulation with 10,000 iterations. Cost-effectiveness acceptability curves were used to present the results of the PSA. As the results are uncertain, it is possible that the wrong decision is made when implementing the most cost-effective strategy based on the current analysis. The expected value of perfect information (EVPI) analysis can be used to assess the expected costs of this decision uncertainty. In this way, the EVPI can be interpreted as the maximum that society should be willing to pay for further evidence to reduce this decision uncertainty. [21] The population EVPI was calculated by multiplying the EVPI per patient by the effective population in the next 10 years (expected life span of the technology) and discounted by a rate of $4 \%$. The Dutch effective population was calculated based on a yearly incidence of 1,743 HER2 positive early breast cancer patients eligible for chemotherapy (based on an annual incidence of 14,070 x 17.7\% HER2+ x 70\% eligible for chemotherapy). [22]

\section{RESULTS}

In the real world scenario and the guideline scenario the trastuzumab treated patient group proved to be more expensive than the group of patients treated with chemotherapy alone. Costs were $€ 243,216$ and $€ 239,657$ for trastuzumab and no trastuzumab for the real world scenario, €224,443 and $€ 218,948$ for the guideline scenario (incremental cost of $€ 3560$ and $€ 5495$, respectively). However, in the trial scenario the no trastuzumab group was more expensive $€ 253,666$ versus $€ 265,116$ (incremental cost of $-€ 11,451$ ). This was also the case for effectiveness in the three scenarios. The incremental QALYs of the real world and the guideline scenarios were 0.827 and 0.861 respectively, while the incremental QALY of the trial scenario was 0.993 . Comparing these groups resulted in ICERs of $€ 4304$, $€ 6382$ and dominance, corresponding to the real world, guideline and trial scenarios. These base case results show that for the real world scenario and the guideline scenario trastuzumab is both more effective (LY and QALY gain) and more costly. In the trial scenario trastuzumab was less costly and more effective (Table 4).

In all three scenarios, the incremental costs could be credited to a high cost of treatment of distant metastases in the no trastuzumab group compared to the trastuzumab

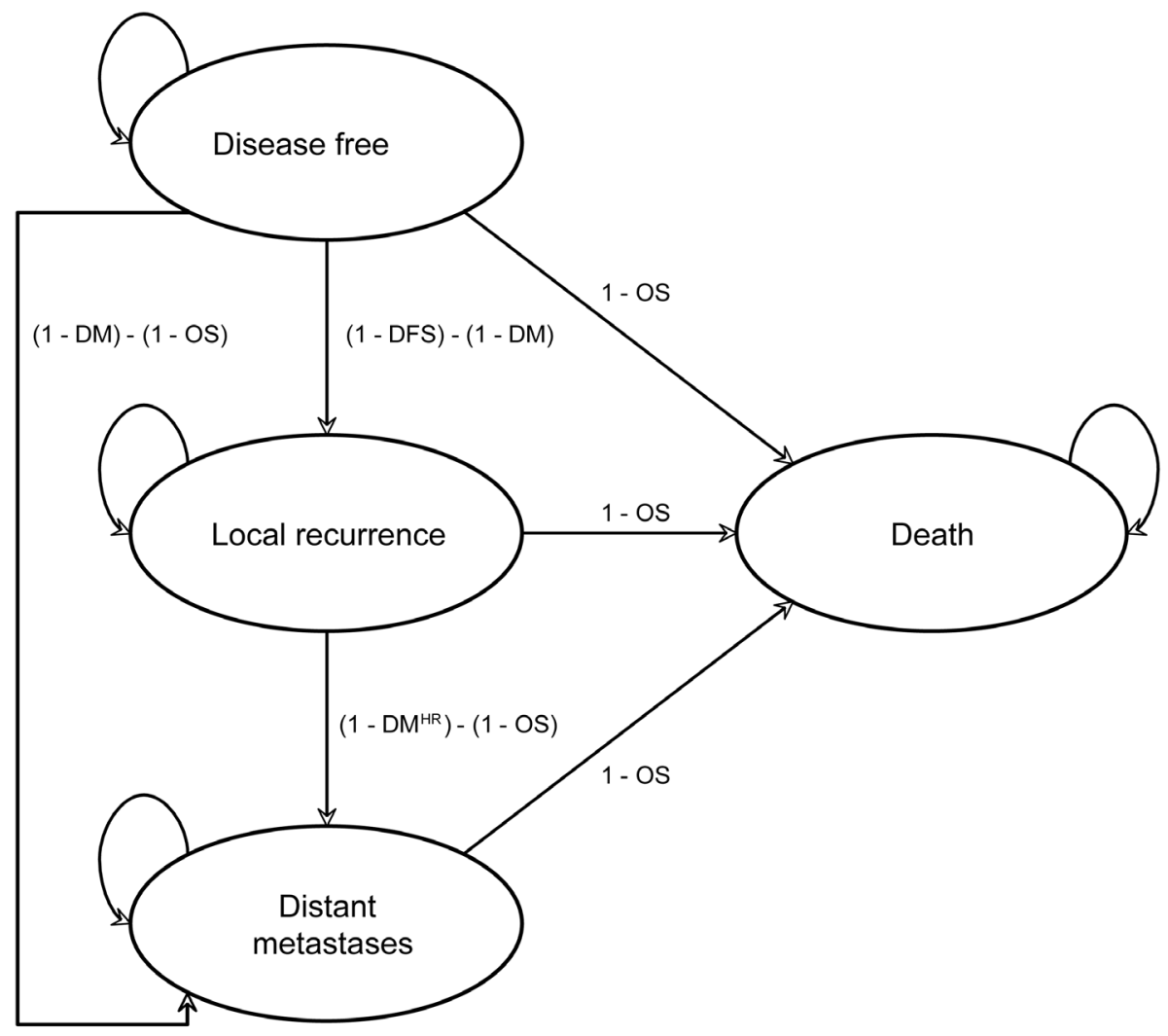

Figure 1: Markov state transition model structure ${ }^{\text {a. }}$ Abbreviations: OS, overall survival; DFS, disease free survival and; DM, distant metastases free survival; HR, hazard ratio for increased risk of distant metastases after local recurrence. ${ }^{\mathrm{a} N o t e}$ that cardiac events are incorporated during the first year only. 
Table 1: The data sources used for parameters in the real world, guideline and trial scenario

\begin{tabular}{|c|c|c|c|}
\hline Scenarios & Real world & Guideline & Trial` $^{\wedge}$ \\
\hline Disease-free survival & Cohort* & Cohort** & HERA trial \\
\hline Overall survival & Cohort* & Cohort** & HERA trial \\
\hline Distant metastases free survival & Cohort* & Cohort** & HERA trial \\
\hline HR Disease-free Survival & \multicolumn{3}{|c|}{ HERA trial } \\
\hline HR Overall survival & \multicolumn{3}{|c|}{ HERA trial } \\
\hline HR Distant metastases & \multicolumn{3}{|c|}{ HERA trial } \\
\hline Duration of treatment benefit & \multicolumn{3}{|c|}{ HERA trial } \\
\hline Probability cardiac event trastuzumab & Cohort* & Cohort* & HERA trial \\
\hline $\mathrm{RR}^{\#}$ cardiac event trastuzumab & \multicolumn{3}{|c|}{ HERA trial } \\
\hline Resource use (other than trastuzumab) & \multicolumn{3}{|c|}{ Cohort ${ }^{*}$} \\
\hline Costs associated with trastuzumab & Cohort* & Cohort $^{* *}$ & Cohort \& HERA trial \\
\hline Health state utiilities & \multicolumn{3}{|c|}{ Cohort $^{*}$} \\
\hline
\end{tabular}

${ }^{\wedge}$ The HERA trial results are published in [10-14]. * Including all HER2 positive patients; ** Patients who received trastuzumab according to the Dutch 2005 guideline.\# Relative Risk.

group. The incremental costs associated with the local recurrence health state were higher in the real world and guideline scenarios compared to the trial scenario. The trial scenario had no costs related to the local recurrence health state. This was caused by the fact that LR is calculated by $1-\mathrm{DFS}$ and $1-\mathrm{DM}$ which in this case is $0 \%$. In our trial scenario 1-DFS $<1$-DM (Table 2).

Similarly to the incremental cost, the incremental QALYs and LYs could be attributed to the higher number of QALYs and LYs generated in the distant metastases health state.

In the one-way sensitivity analyses the ICERs were sensitive to the duration of the treatment effect (Table 5). In addition, Table 6 indicates that assuming treatment dependent health state costs would result in dominance of trastuzumab in all three scenarios.
At a willingness to pay (WTP) threshold of $€ 80,000$ per QALY gained, the probability that trastuzumab treatment is cost-effective is $99 \%$ or higher in all three scenarios (Figure 2). The expected value of perfect information (EVPI) analysis, at a threshold of $€ 80,000$ per QALY, amounts to $€ 477,766, € 486,041$ and $€ 1,346$, respectively in the real world, guideline and trial scenario.

\section{DISCUSSION}

In this study, we determined the costs and costeffectiveness of one year of adjuvant trastuzumab treatment in early HER2-positive breast cancer versus chemotherapy alone. We assessed three scenarios. We found that the ICER in the real world scenario was $€ 4,304$ per QALY gained, in the guideline scenario the

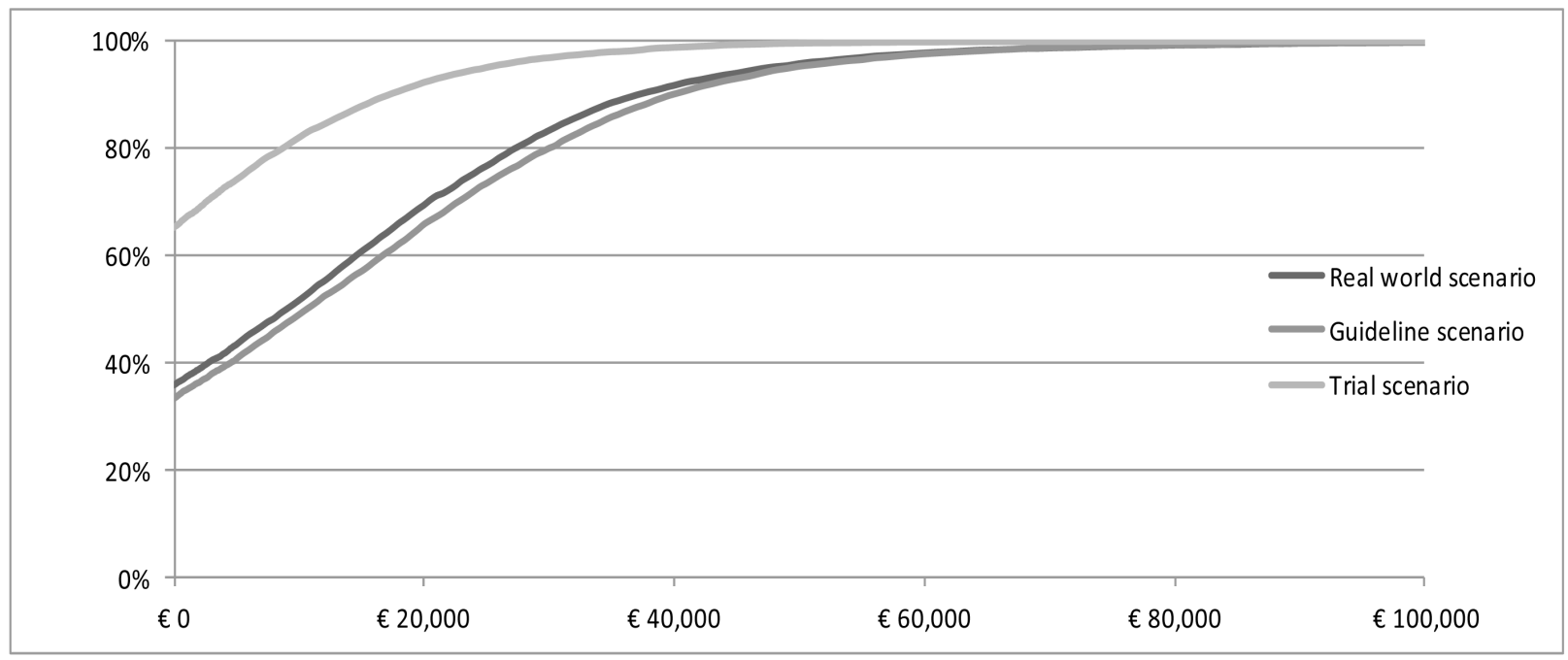

Figure 2: CEAC. Cost-effectiveness acceptability curve of trastuzumab in real world scenario, guideline scenario and trial scenario. 
Table 2: Transition probabilities used in the model in the three scenarios

\begin{tabular}{|c|c|c|c|c|}
\hline $\begin{array}{l}\text { Transition probabilities } \\
\text { Real world scenario - } \\
\text { trastuzumab }\end{array}$ & Estimate & $\begin{array}{r}\text { Standard } \\
\text { Error }\end{array}$ & Distribution & Source \\
\hline 1-Overall Survival, first year* & 0.000 & - & fixed & Cohor \\
\hline 1-Overall Survival , > first year* & 0.026 & 0.006 & Beta & Cohor \\
\hline $\begin{array}{l}\text { 1-Disease Free Survival, first } \\
\text { year }\end{array}$ & 0.016 & 0.031 & Beta & Cohor \\
\hline $\begin{array}{l}\text { 1-Disease Free Survival, > first } \\
\text { year }\end{array}$ & 0.054 & 0.067 & Beta & Cohor \\
\hline 1-Distant Metastases, first year & 0.003 & 0.002 & Beta & Cohor \\
\hline 1-Distant Metastases, $>$ first year & 0.041 & 0.006 & Beta & Cohor \\
\hline $\begin{array}{l}\text { Transition probabilities } \\
\text { Guideline scenario - trastuzumab }\end{array}$ & Estimate & $\begin{array}{r}\text { Standard } \\
\text { Error }\end{array}$ & Distribution & Source \\
\hline 1-Overall Survival, first year* & 0.000 & - & fixed & Cohort \\
\hline 1-Overall Survival , > first year* & 0.029 & 0.006 & Beta & Cohort \\
\hline $\begin{array}{l}\text { 1-Disease Free Survival, first } \\
\text { year }\end{array}$ & 0.019 & 0.009 & Beta & Cohor \\
\hline $\begin{array}{l}\text { 1-Disease Free Survival, > first } \\
\text { year }\end{array}$ & 0.052 & 0.006 & Beta & Cohor \\
\hline 1-Distant Metastases, first year & 0.004 & 0.003 & Beta & Cohort \\
\hline 1-Distant Metastases, > first year & 0.048 & 0.007 & Beta & Cohort \\
\hline $\begin{array}{l}\text { Transition probabilities } \\
\text { Trial scenario - trastuzumab }\end{array}$ & Estimate & $\begin{array}{r}\text { Standard } \\
\text { Error }\end{array}$ & Distribution & Source \\
\hline 1-Overall Survival, first year* & 0.023 & 0.001 & Beta & HERA[13] \\
\hline 1-Overall Survival , > first year* & 0.023 & 0.001 & Beta & HERA[13] \\
\hline $\begin{array}{l}\text { 1-Disease Free Survival, first } \\
\text { year }\end{array}$ & 0.042 & 0.002 & Beta & HERA[13] \\
\hline $\begin{array}{l}\text { 1-Disease Free Survival, > first } \\
\text { year }\end{array}$ & 0.042 & 0.002 & Beta & HERA[13] \\
\hline 1-Distant Metastases, first year & 0.050 & 0.004 & Beta & HERA[11] \\
\hline 1-Distant Metastases, > first year & 0.050 & 0.004 & Beta & HERA[11] \\
\hline $\begin{array}{l}\text { Transition probabilities } \\
\text { All scenarios }\end{array}$ & Estimate & $\begin{array}{r}\text { Standard } \\
\text { Error (HR) }\end{array}$ & Distribution & Source \\
\hline HR Overall Survival & 0.760 & 0.077 & LogNormal & HERA[13] \\
\hline HR Disease Free Survival & 0.760 & 0.064 & LogNormal & HERA[13] \\
\hline HR Distant Metastases & 0.600 & 0.102 & LogNormal & HERA[11] \\
\hline $\begin{array}{l}\text { HR for increased risk of distant } \\
\text { metastases after local recurrence }\end{array}$ & 3.640 & 0.300 & LogNormal & HERA[31] \\
\hline $\begin{array}{l}\text { Duration of treatment benefit } \\
\text { (years) }\end{array}$ & 8.000 & 0.307 & LogNormal & HERA[13] \\
\hline
\end{tabular}

*In case OS is smaller than age related mortality based on Dutch all-cause mortality for women, the age related mortality is used

In all three scenarios the HR for the group not treated with trastuzumab was calculated using the inverse of the HR of the HERA trial. 
Table 3: Total health state costs and utility scores per year

\begin{tabular}{|c|c|c|c|c|}
\hline Cost input parameters & Estimate & $\begin{array}{l}\text { Standard } \\
\text { Error }\end{array}$ & Distribution & Source \\
\hline \multicolumn{5}{|l|}{ Health state costs (per year) } \\
\hline Disease Free Survival, first year & $€ 12,776$ & $€ 798.14$ & Gamma & Cohort \\
\hline $\begin{array}{l}\text { Disease Free Survival, > first } \\
\text { year }\end{array}$ & $€ 1,237$ & $€ 107.49$ & Gamma & Cohort \\
\hline Local recurrence, first year & $€ 12,777$ & $€ 2,203.85$ & Gamma & Cohort \\
\hline Local recurrence, $>$ first year & $€ 14,149$ & $€ 4,679.22$ & Gamma & Cohort \\
\hline Distant Metastases, first year & $€ 30,165$ & $€ 2,339.46$ & Gamma & Cohort \\
\hline Distant Metastases, > first year & $€ 47,959$ & $€ 13,877.79$ & Gamma & Cohort \\
\hline Cardiac Monitoring, first year & $€ 467$ & $€ 18.90$ & Gamma & Cohort \\
\hline $\begin{array}{l}\text { Trastuzumab \# vials } 150 \mathrm{mg} \text { per } \\
\text { cycle }\end{array}$ & 3 & 0.050 & Gamma & Cohort \\
\hline Trastuzumab \# administrations & 15 & 0.420 & Gamma & Cohort \\
\hline Trastuzumab cost / vial $150 \mathrm{mg}$ & $€ 605$ & & Fixed & \\
\hline Costs of administration (day care) & $€ 257$ & - & Fixed & \\
\hline Health state utility scores & Estimate & $\begin{array}{l}\text { Standard } \\
\text { Error }\end{array}$ & Distribution & Source \\
\hline Disease free survival, first year & 0.728 & 0.016 & Beta & Cohort \\
\hline Disease free survival, $>$ first year & 0.805 & 0.021 & Beta & Cohort \\
\hline Local recurrence, first year & 0.725 & 0.021 & Beta & Cohort \\
\hline Local recurrence, $>$ first year & 0.708 & 0.088 & Beta & Cohort \\
\hline Distant metastases, first year & 0.584 & 0.063 & Beta & Cohort \\
\hline Distant metastases, $>$ first year & 0.604 & 0.046 & Beta & Cohort \\
\hline Cardiac toxicity & 0.600 & 0.010 & Beta & [19] \\
\hline Disutility & 0.128 & 0.019 & & \\
\hline
\end{tabular}

*treatment costs were assumed to be fixed since differences in these costs are most likely a result of variability (not parameter uncertainty)

ICER was $€ 6,382$ per QALY gained and finally the trial scenario showed dominance for trastuzumab. Based on these results, we conclude that trastuzumab in early breast cancer is a cost-effective intervention in all three scenarios in the Netherlands. Very little to no uncertainty was seen.

Reports from several countries have shown that adjuvant trastuzumab in early breast cancer patients is cost-effective. Previously, two reviews showed modelled cost-effectiveness analyses based on data from clinical trials in patients with HER2-positive early breast cancer, treated with adjuvant trastuzumab. Our study is one of the few studies that included real world data in a costeffectiveness analysis.

In our results, the trial scenario showed dominance, while even though the real world scenario showed trastuzumab to be cost effective, there was no dominance. This difference was due to higher cost in the distant metastases group in the trastuzumab group versus the no trastuzumab group. Before the introduction of trastuzumab, HER2 positive patients with metastatic disease had a very poor prognosis, the introduction of trastuzumab made the prognosis better, but was also more costly.

The systematic review from Chan et al. selected thirteen articles that included cost-effectiveness analyses of 10 countries. The cost-effectiveness ratios ranged from $\$ 5020 /$ QALY to $\$ 134,610 /$ QALY. Most studies reported a favorable cost-effectiveness. [23] Mc-Keage et al also performed a review, this study reported that trastuzumab was cost-effective from a healthcare payer or societal perspective in several countries. Incremental costs per QALY or life-year gained with trastuzumab administered subsequent to or concurrent with chemotherapy compared with chemotherapy alone were consistently within accepted local thresholds for cost effectiveness. [24]

Our trial scenario was based on the HERA trial. We based our DFS data on the 8-year FU and our DM data from the 2 year FU, since the 8year FU did not 
Table 4: Base case results for all three scenarios

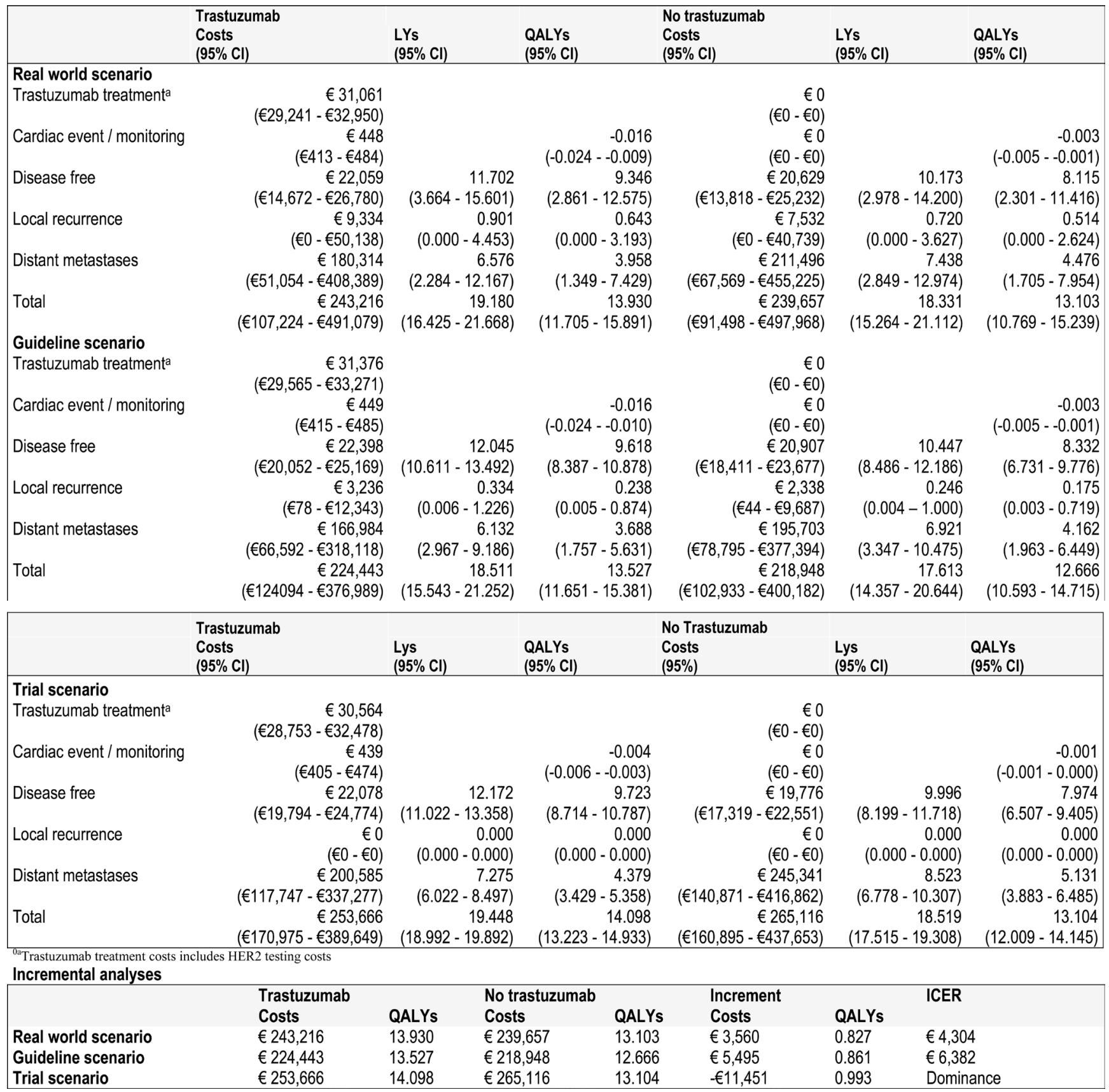

mention DM data. Reviews have shown that trastuzumab is considered cost-effective when using the HERA trial data, but also when using other trials including FinHER trial, NSABP B-31 and NCCTG N9831 trials. For example Dedes et al., a model-based study from Swiss healthcare perspective, showed that adjuvant trastuzumab proved to be cost-effective based on the data of the HERA trial and based on the data of the FinHER study. [2] Garisson et al. concluded the same based on data of the NSABP B-31 and NCCTG N9831trials. [3] Likewise cost-effectiveness of trastuzumab was seen in different (developed) countries, next to Switzerland, also USA (ICER of 39,982 US dollars per QALY) [4], Italy (ICER of 14,861 US dollars) [25] and Norway (estimated ICER of $€ 44,934$ per QALY) [26]. In a Belgian study, a HERA trial scenario was compared to a FinHER scenario. One year trastuzumab was shown to be cost-effective although never cost saving due to the higher initial treatment costs, whereas the 9-week FinHER regimen was cost saving. [6] So, most studies suggest that trastuzumab may be cost-effective for treatment of early breast cancer in a 1-year treatment regimen, based on the frequently proposed threshold of 50.000 US dollar / QALY. [27, 28] This is comparable to the proposed Dutch threshold of $€ 80,000$.

To our knowledge, only one study discussed real world cost-effectiveness of trastuzumab. The study of 
Table 5: Sensitivity analysis; assuming $4 y$ treatment effect duration (instead of 8y) - probabilistic

\begin{tabular}{|l|c|c|c|c|c|c|c|}
\hline & \multicolumn{2}{|c|}{ Trastuzumab } & \multicolumn{2}{c|}{ No trastuzumab } & \multicolumn{2}{c|}{ Increment } & ICER \\
\hline & Costs & QALYs & Costs & QALYs & Costs & QALYs & \\
\hline Real world scenario & $€ 242,295$ & 13.938 & $€ 226,954$ & 13.554 & $€ 15,341$ & 0.384 & $€ 39,934$ \\
\hline Guideline scenario & $€ 226,014$ & 13.545 & $€ 210,056$ & 13.142 & $€ 15,957$ & 0.402 & $€ 39,655$ \\
\hline Trial scenario & $€ 253,494$ & 14.098 & $€ 249,955$ & 13.547 & $€ 3,539$ & 0.552 & $€ 6,412$ \\
\hline
\end{tabular}

Table 6: Sensitivity analysis; assuming treatment dependent DM health state costs - probabilistic

\begin{tabular}{|l|c|c|c|c|c|c|c|}
\hline & \multicolumn{2}{|c|}{ Trastuzumab } & \multicolumn{2}{c|}{ No trastuzumab } & \multicolumn{2}{c|}{ Increment } & ICER \\
\hline & Costs & QALYs & Costs & QALYs & Costs & QALYs & \\
\hline Real world scenario & $€ 176,541$ & 13.927 & $€ 315,715$ & 13.097 & $-€ 139,175$ & 0.830 & Dominance \\
\hline Guideline scenario & $€ 165,256$ & 13.551 & $€ 293,593$ & 12.686 & $-€ 128,337$ & 0.865 & Dominance \\
\hline Trial scenario & $€ 181,133$ & 14.095 & $€ 357,625$ & 13.103 & $-€ 176,492$ & 0.993 & Dominance \\
\hline
\end{tabular}

Hedden et al. indicated that trastuzumab is cost-effective in a real world setting. In this Canadian context the cost per QALY gained was $\$ 13,095$. [29]

Differences in ICER between the diverse studies could be explained by country-specific differences in health care systems, costs, modeling concepts and underlying assumptions. This underpins the necessity of country-specific cost-effectiveness analysis. Another argument for different ICERs between studies is the fact that the longer the follow up, the more cross over there is in patients that were still treated with trastuzumab, while originally at the start of the clinical trials these patient were included in the no trastuzumab group.

The 8-year follow up data from the HERA trial showed that the trastuzumab versus no trastuzumab groups were contaminated by a $52.1 \%$ crossover. [13] On the other hand, real world data is virtually always biased by confounding by indication, and hence any estimate of comparative effectiveness. In our study we used the relative effectiveness from the trials, therefore there could be no confounding by indication. If we had used relative effect of the real world data we would have had confounding by indication. As a result, the estimation of (longer-term) comparative effectiveness is challenged, whether trial data or real world data are used. Massive crossover in trials may lead to an underestimation of treatment effect, while confounding by indication is mostly thought to overestimate treatment effect, and methods are developed to correct for both types of biases. [30] As all three scenarios based comparative effectiveness on the HERA trial, the reported ICERs may be overestimations. In health policy decision making trial and real world evidence are complementary, and further research should focus on ways to combine and handle bias in both sources of evidence.

Our study was unique in comparing real world data to a trial data and a guideline scenario. Selecting patients according to the 2005 guideline (guideline scenario) leads to the highest ICER $(€ 6,382)$, and both the real world and the guideline scenario result in higher ICERs than the trial scenario. These results confirm that analyses based on data from clinical trials contain the most beneficial scenario. In addition, a trend is seen that when a breakthrough product is released, there is also a fast implementation, causing the real world to be a step ahead of the guidelines. In this study the real world scenario is more cost-effective than the guideline scenario.

In conclusion, adjuvant trastuzumab in the real world can be considered cost-effective in all three scenarios, but most cost-effective when all input is based on trial data.

\section{CONFLICTS OF INTEREST}

There is no conflict of interest.

\section{FUNDING}

This work was supported by Netherlands Organization for Health Research and Development (ZonMw: 80-82500-98-9056) and Roche Netherlands B.V.

\section{REFERENCES}

1. Essers BA, Seferina SC, Tjan-Heijnen VC, Severens JL, Novak A, Pompen M, Oron UH, Joore MA. Transferability of model-based economic evaluations: the case of trastuzumab for the adjuvant treatment of HER2-positive early breast cancer in the Netherlands. Value in health. 2010; 13:375-80.

2. Dedes KJ, Szucs TD, Imesch P, Fedier A, Fehr MK, Fink D. Cost-effectiveness of trastuzumab in the adjuvant treatment of early breast cancer: a model-based analysis of the HERA and FinHer trial. Ann Oncol. 2007; 18:1493-99.

3. Garrison LP Jr, Lubeck D, Lalla D, Paton V, Dueck A, Perez EA. Cost-effectiveness analysis of trastuzumab in the adjuvant setting for treatment of HER2-positive breast cancer. Cancer. 2007; 110:489-98.

4. Kurian AW, Thompson RN, Gaw AF, Arai S, Ortiz R, Garber AM. A cost-effectiveness analysis of adjuvant trastuzumab regimens in early HER2/neu-positive breast 
cancer. J Clin Oncol. 2007; 25:634-41.

5. Millar JA, Millward MJ. Cost effectiveness of trastuzumab in the adjuvant treatment of early breast cancer: a lifetime model. Pharmacoeconomics. 2007; 25:429-42.

6. Neyt M, Huybrechts M, Hulstaert F, Vrijens F, Ramaekers D. Trastuzumab in early stage breast cancer: a costeffectiveness analysis for Belgium. Health Policy. 2008; 87:146-59.

7. Hall PS, Hulme C, McCabe C, Oluboyede Y, Round J, Cameron DA. Updated cost-effectiveness analysis of trastuzumab for early breast cancer: a UK perspective considering duration of benefit, long-term toxicity and pattern of recurrence. Pharmacoeconomics. 2011; 29:41532.

8. Garrison LP Jr, Neumann PJ, Erickson P, Marshall D, Mullins CD. Using real-world data for coverage and payment decisions: the ISPOR Real-World Data Task Force report. Value in health. 2007; 10:326-35.

9. Seferina SC, Lobbezoo DJ, de Boer M, Dercksen MW, van den Berkmortel F, van Kampen RJ, van de Wouw AJ, de Vries B, Joore MA, Peer PG, Voogd AC, Tjan-Heijnen VC. Real-Life Use and Effectiveness of Adjuvant Trastuzumab in Early Breast Cancer Patients: A Study of the Southeast Netherlands Breast Cancer Consortium. Oncologist. 2015; 20:856-63.

10. Piccart-Gebhart MJ, Procter M, Leyland-Jones B, Goldhirsch A, Untch M, Smith I, Gianni L, Baselga J, Bell R, Jackisch C, Cameron D, Dowsett M, Barrios CH, et al, and Herceptin Adjuvant (HERA) Trial Study Team. Trastuzumab after adjuvant chemotherapy in HER2positive breast cancer. N Engl J Med. 2005; 353:1659-72.

11. Smith I, Procter M, Gelber RD, Guillaume S, Feyereislova A, Dowsett M, Goldhirsch A, Untch M, Mariani G, Baselga J, Kaufmann M, Cameron D, Bell R, et al. 2-year followup of trastuzumab after adjuvant chemotherapy in HER2positive breast cancer: a randomised controlled trial. Lancet. 2007; 369:29-36.

12. Gianni L, Dafni U, Gelber RD, Azambuja E, Muehlbauer S, Goldhirsch A, Untch M, Smith I, Baselga J, Jackisch C, Cameron D, Mano M, Pedrini JL, et al. Treatment with trastuzumab for 1 year after adjuvant chemotherapy in patients with HER2-positive early breast cancer: a 4-year follow-up of a randomised controlled trial. Lancet Oncol. $2011 ; 12: 236-44$.

13. Goldhirsch A, Gelber RD, Piccart-Gebhart MJ, de Azambuja E, Procter M, Suter TM, Jackisch C, Cameron D, Weber HA, Heinzmann D, Dal Lago L, McFadden E, Dowsett M, et al. 2 years versus 1 year of adjuvant trastuzumab for HER2-positive breast cancer (HERA): an open-label, randomised controlled trial. Lancet. 2013; 382:1021-28.

14. Suter TM, Procter M, van Veldhuisen DJ, Muscholl M, Bergh J, Carlomagno C, Perren T, Passalacqua R, Bighin C,
Klijn JG, Ageev FT, Hitre E, Groetz J, et al. Trastuzumabassociated cardiac adverse effects in the herceptin adjuvant trial. J Clin Oncol. 2007; 25:3859-65.

15. C.B.o.S. http://statline.cbs.nl/StatWeb/publication/?DM $=\mathrm{SLNL} \& \mathrm{PA}=71311$ ned $\& \mathrm{D} 1=0-1,4-5 \& \mathrm{D} 2=0 \& \mathrm{D} 3=$, $51,64,77,90,103,116,129,142,155,168,181,194,219,232,1 \&$ $\mathrm{HDR}=\mathrm{G} 1, \mathrm{~T} \& \mathrm{STB}=\mathrm{G} 2 \& \mathrm{CHARTTYPE}=1 \& \mathrm{VW}=\mathrm{T}$.

16. CVZ Fk. Z-index taxe. [Article in Dutch]. Farmacotherapeutisch kompas]. 2009.

17. Oostenbrink J, et al. Handleiding voor kostenonderzoek, methoden en standaard kostprijzen voor economische evaluaties in de gezondheidszorg. [Article in Dutch]. College voor Zorgverzekeringen. 2004.

18. Dolan Kind PG, Williams A. A social tariff for EuroQol: results from a UK general population survey, discussion paper no 138. Centre for Health Economics, University of York, York. 1995.

19. Calvert MJ, Freemantle N, Cleland JG. The impact of chronic heart failure on health-related quality of life data acquired in the baseline phase of the CARE-HF study. Eur J Heart Fail. 2005; 7:243-51.

20. 2006. RvVeZZedz. Raad voor Volksgezondheid en Zorg. Zinnige en duurzame zorg. [Article in Dutch]. 2006.

21. Briggs A, Claxton K, Sculpher MJ. Decision modelling for health economic evaluation2006, Oxford: Oxford University Press. Decision modelling for health economic evaluation 2006, Oxford: Oxford University Press. Oxford: Oxford University Press; 2006.

22. http://www.cijfersoverkanker.nl/selecties/incidentie borstkanker/img521b5d17e8d90?row=0\&direction=down \#table 2013. [cited 2013 27-08-2013.].

23. Chan AL, Leung HW, Lu CL, Lin SJ. Cost-effectiveness of trastuzumab as adjuvant therapy for early breast cancer: a systematic review. Ann Pharmacother. 2009; 43:296-303.

24. McKeage K, Lyseng-Williamson KA. Trastuzumab: a pharmacoeconomic review of its use in early breast cancer. Pharmacoeconomics. 2008; 26:699-719.

25. Liberato NL, Marchetti M, Barosi G. Cost effectiveness of adjuvant trastuzumab in human epidermal growth factor receptor 2-positive breast cancer. J Clin Oncol. 2007; 25:625-33.

26. Norum J, Olsen JA, Wist EA, Lønning PE. Trastuzumab in adjuvant breast cancer therapy. A model based costeffectiveness analysis. Acta Oncol. 2007; 46:153-64.

27. Laupacis A, Feeny D, Detsky AS, Tugwell PX. How attractive does a new technology have to be to warrant adoption and utilization? Tentative guidelines for using clinical and economic evaluations. CMAJ. 1992; 146:47381.

28. Evans C, Tavakoli M, Crawford B. Use of quality adjusted life years and life years gained as benchmarks in economic evaluations: a critical appraisal. Health Care Manage Sci. 2004; 7:43-49. 
29. Hedden L, O'Reilly S, Lohrisch C, Chia S, Speers C, Kovacic L, Taylor S, Peacock S. Assessing the real-world cost-effectiveness of adjuvant trastuzumab in HER-2/neu positive breast cancer. Oncologist. 2012; 17:164-71.

30. Ahrens W, et al. Handbook of Epidemiology. 2007. 